\title{
Towards seamless computing and metacomputing in Java
}

\author{
DENIS CAROMEL*, WILFRIED KLAUSER AND JULIEN VAYSSIÈRE \\ INRIA Sophia Antipolis, CNRS - I3S - Univ. Nice Sophia Antipolis, BP 93, 06902 Sophia Antipolis Cedex, France \\ (e-mail: First.Last@sophia.inria.fr) \\ http://www.inria.fr/sloop/javall/
}

\section{SUMMARY}

Due to its platform-independent execution model, its support for networking, multithreading and mobile code, Java has given hope that easy Internet-wide high-performance network computing was at hand. Numerous attempts have then been made at providing a framework for the development of such metacomputing applications. Unfortunately, none of them addresses seamless sequential, multithreaded and distributed computing, i.e. the execution of the same application on a multiprocessor shared-memory machine as well as on a network of workstations, or on any hierarchical combination of both. In this paper we first identify four requirements for the development of such metacomputing frameworks. We then introduce Java// (pronounced Java Parallel), a $\mathbf{1 0 0 \%}$ Java library that provides transparent remote objects as well as asynchronous two-way calls, high reuse potential and high-level synchronization mechanisms. We also present the metaobject protocol (MOP) Java// is built on and describe a distributed collaborative raytracing test application built using Java//. @ 1998 John Wiley \& Sons, Ltd.

\section{INTRODUCTION}

In order to provide a framework for the development of cross-paradigm metacomputing environments [1-3] within the scope of the Java language[4] and environment[5], we identify four key requirements: polymorphism between local and remote objects, high-level synchronization mechanisms, reuse of sequential code and the availability of a $100 \%$ Java portable library.

\subsection{Transparent remote objects}

First, let us focus on cross-paradigm portability. Cross-platform portability is genuinely achieved by the standard Java execution environment. An application written in Java is compiled into an architecture-neutral bytecode format, which then executes on a Java Virtual Machine (JVM) whose purpose is to hide the nature of the underlying platform.

Some JVM implementations provide access to native threads, which, when run on a multiprocessor machine, permits automatic mapping of Java threads onto the set of available processors. This feature abolishes the frontier between a monoprocessor machine and a multiprocessor, shared-memory machine when it comes to executing multithreaded Java applications. It results in instant speedup for applications built around concurrent activities, provided there actually is some parallelism between the threads. Consequently, code reuse for porting Java threaded applications from a monoprocessor machine to a multiprocessor machine is not an issue since the application code for a monoprocessor

${ }^{*}$ Correspondence to: Denis Caromel, INRIA Sophia-Antipolis, BP 93, 06902 Sophia-Antipolis Cedex, France. 


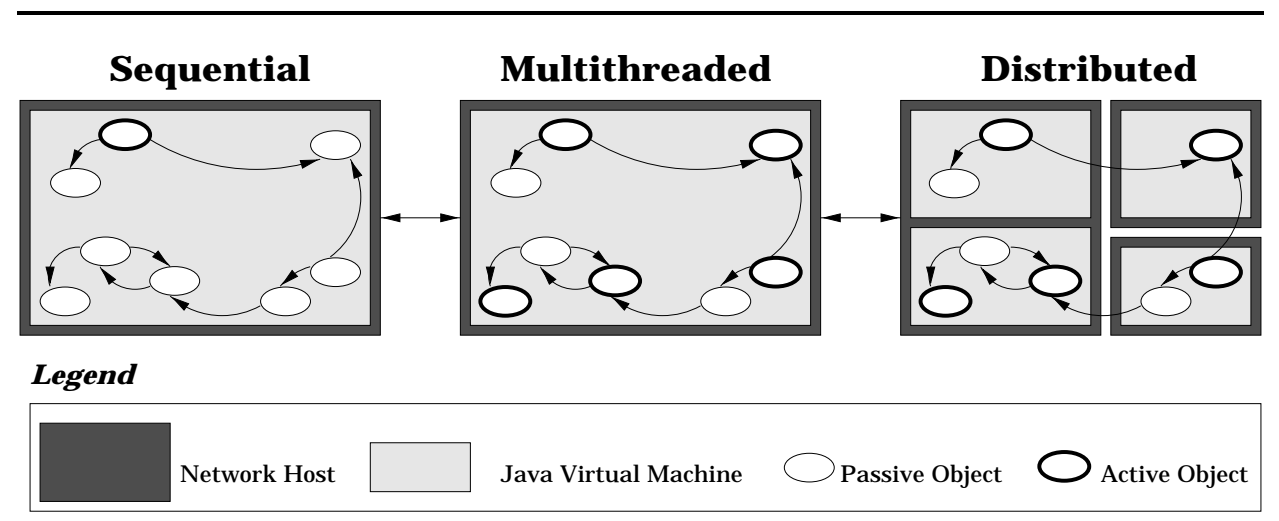

Figure 1. Seamless sequential, multithreaded and distributed programming

machine does not need any modification at all to run on a multiprocessor shared-memory machine.

Nevertheless, a huge gap yet exists between multithreaded and distributed Java applications which forbids code reuse in order to build distributed applications from multithreaded applications (see Figure 1). Both JavaRMI and JavaIDL, as examples of distributed object libraries in Java, put a heavy burden on the programmer because they require deep modifications of existing code in order to turn local objects into remote-accessible ones.

In these systems, remote objects need to be accessed through some specific interfaces. One could argue that programming to an interface is usually considered as a better practice than programming to an implementation. This is undoubtedly true, but the core of the problem is that implementation classes are forced to move from one place in the inheritance graph to another in order to become remote-accessible classes. Method signatures also need to be modified in order to throw distribution-related exceptions, which does not allow a clear separation of concerns between functional code and distribution-related code.

As a consequence, these distributed objects libraries do not allow polymorphism between local and remote objects. This feature is our first requirement for a metacomputing framework. It is strongly required in order to let the programmer concentrate first on modeling and algorithmic issues rather than lower-level tasks such as object distribution, mapping and load balancing.

\subsection{High-level synchronization mechanism}

Our second requirement for metacomputing is higher-level synchronization mechanisms. Although monitor-like primitives[6] found in Java may be theoretically sufficient for expressing synchronization, implementing complex synchronization specifications using such low-level tools is definitely cumbersome and error-prone. Moreover, such architectures do not scale well and have some reuse problems[7]. Such an architecture also assumes a shared memory of some kind, which does not fit well in a system that needs to address distribution as well.

\subsection{Reuse of sequential code}

Reuse of sequential code does not here mean reusing legacy applications in order to build concurrent and distributed Java applications. It is our opinion that sequential code should no 
more be considered as a single-threaded implementation of a problem but rather as a highlevel expression of the fundamental abstractions and operations in a system. In this context, code reuse simply means adapting the sequential code to a particular metacomputing environment. This includes, but is not limited to, deciding which objects should have their own activity (thread) and how they should be distributed over a set of computing nodes.

When designing an object-oriented application, the programmer usually starts with creating high-level domain-dependent abstractions and turns these into objects and classes. These classes and objects are then connected together using inheritance, composition, or any other technique, which eventually results in a modeling of the domain managed by the application.

Deciding which objects should have an activity on their own or distributing objects over several address spaces is definitely a lower-level issue. As a matter of fact, object distribution or the expression of parallel activities is always constrained by the actual system the application should be implemented on. This is why we believe a framework for metacomputing applications should provide a clear separation between high-level application design and lower-level implementation issues such as object distribution or managing concurrent activities, and postpone as much as possible deciding which objects should be active.

\subsection{A portable, non-intrusive library}

A rather large number of research projects have already been conducted on transparent remote objects in Java[8,9]. Two major implementation techniques are used: some change the Java Virtual Machine or the Java-to-bytecode compiler; others rely on some source preprocessing. These techniques lead to two different flaws. The first one fails at providing Internet-wide portability by requiring installation of a specific runtime environment on each possible node of the computation. The second one requires that the programmer has access to the source code of the objects he wants to make remote, which is barely never the case when using third-party libraries. Consequently, a library that aims at distributing Java objects transparently has to be $100 \%$ Java and only require access to the compiled representation of classes, not to the sources.

\section{THE JAVA// FRAMEWORK}

In order to meet these requirements, we have designed and implemented Java// (pronounced Java Parallel), a Java library for seamless sequential, multithreaded and distributed programming.

Java// only consists of a collection of $100 \%$ Java classes, thus requiring no change to the standard Java execution environment. The Java// model uses by default the following principles:

- heterogeneous model with both passive and active objects (threads, actors)

- sequential processes

- unified syntax between message passing and inter-process communication

- systematic asynchronous communications towards active objects

- wait-by-necessity (automatic and transparent futures)

- automatic continuations (a transparent delegation mechanism)

- no shared passive objects (call-by-value between processes)

C191998 John Wiley \& Sons, Ltd.

Concurrency: Pract. Exper., 10, 1043-1061 (1998) 
- centralized and explicit control by default

- polymorphism between standard objects, active objects, and remote objects.

\subsection{Model of execution}

Given a standard Java object, there are several new behaviors we would like to give it transparently: location transparency, activity transparency and advanced synchronization mechanism.

Location transparency provides polymorphism between local and remote objects. Activity transparency hides the fact that methods invoked on an active object actually execute in a separate thread. This is achieved using transparent future objects and wait-by-necessity[10]. Advanced synchronization mechanisms allow an easy and safe implementation of potentially complex synchronization policies.

Let us have a look at how these different features can be obtained within the scope of the Java language.

In most distributed objects systems, such as RMI or CORBA, location transparency is achieved using the proxy pattern[11]. A local object (the so-called proxy) acts as a representative for an object that resides in another address space, possibly on another machine across a network. This proxy encapsulates all communication details so that other local objects do not know they are actually sending messages to a remote object.

Adding a new behavior to an object, such as its own thread of execution, may usually be achieved using two different object-oriented techniques: multiple inheritance and composition. Multiple inheritance allows effortless extension of a class behavior, provided these different behaviors are quite orthogonal, like functional code and synchronization, for example. The composition mimics a complex object by delegating different behaviors to different objects.

As Java features simple class inheritance and multiple interface inheritance, we have chosen to take the best from both worlds. We use composition for implementation of multiple behaviors, while multiple interface inheritance is used for declaring these behaviors.

In Java//, any standard object (Figure 2 (top)) may be extended through composition with a pair of objects: a proxy and a body (Figure 2 (bottom)). The active object is actually composed of two objects: the body and the standard object. In terms of metaobjects, the proxy transparently reifies method invocations. Method invocations are 'trapped' and

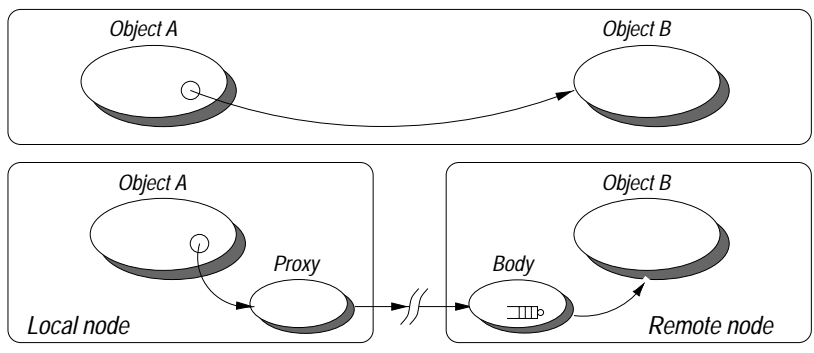

Figure 2. Standard and Javall models of execution contrasted 
converted into instances of the MethodCall class. These method invocations may then be manipulated as first-class objects in order to implement any new semantics. The body receives these reified calls and stores them into a queue of pending calls. It then executes them in an order specified by a given synchronization policy. If none is provided, the body defaults to a FIFO behavior.

The programmer has the possibility to override the default FIFO-ordered policy by writing code for explicitly managing the queue of pending calls on an object. This gives him total control over the synchronization strategy. Note that any other synchronization abstraction may be implemented using the explicit one, such as, for example, an implicit one where the programmer declares a set of properties that constrains the default FIFO-ordered policy.

\subsection{Programming active objects}

Given a sequential Java program, it takes only minor modifications from the programmer to turn it into a multithreaded, ready-for-metacomputing program. We first focus on active object creation and then discuss inter-object synchronization.

\subsubsection{Active object creation}

Java// actually only requires instantiation code to be modified in order to transform a standard object into an active one. All the code that had previously been written for the passive version of the same object remains perfectly valid. Besides the standard constructor parameters for the object, the creation of an active object requires at least the node to create the object on (represented by an instance of class Mapping). Depending on special semantics requirements, additional parameters may be passed.

Here is a sample of code with several techniques for turning a passive instance of class A into an active, possibly remote, one. A standard object created through such a statement:

A $a=$ new A ("foo", 7);

becomes

\section{- instantiation-based}

Object [] params $=\{$ "foo", new Integer (7) $\}$;

A $\mathrm{a}=$ (A) Javall.newActive ("A", params, myMapping);

\section{- or class-based}

class pA extends A implements Active \{\}

Object [] params $=\{$ foo", new Integer (7) $\}$;

$\mathrm{A} a=$ (A) Javall.newActive ("pA", params, myMapping);

- or object-based

A $a=$ new A ("foo", 7);

$\mathrm{a}=$ (A) Javall.turnActive (a, myMapping);

CC1998 John Wiley \& Sons, Ltd.

Concurrency: Pract. Exper., 10, 1043-1061 (1998) 
This piece of code creates an instance of class A or pA on node myMapping. The mapping mechanism between nodes and actual virtual machines, processors and network hosts will be described later. The active instance just created owns its own thread that executes methods invoked on this object in a default FIFO order. The semantics of calls to such an object are transparently asynchronous, with no code modification being required on the caller's side. This sample also illustrates instantiation-based reification (see Section 4) contrasted with class-based reification and object-based reification.

- Instantiation-based reification is mostly a convenience technique. It allows the programmer to create an active instance of A with a FIFO behavior without defining any new class.

- Class-based reification is the core of Java//'s philosophy. Given a class A, the programmer writes a subclass $\mathrm{pA}$ that inherits directly from $\mathrm{A}$ and implements a specific interface such as Active. He or she may also provide a live method in class pA for giving a specific activity or managing synchronization, as we see in Section 2.3

- Object-based reification makes use of the Javall.turnActive method, which enables us to attach an active behavior to an existing object at any time after its creation. This is especially useful when we do not have access to the code that creates the standard object we want to make active.

We suggest the use of the factory method pattern[11] in order to nicely encapsulate the code needed to instantiate active objects. This would result in a static method newActive in class $\mathrm{pA}$ :

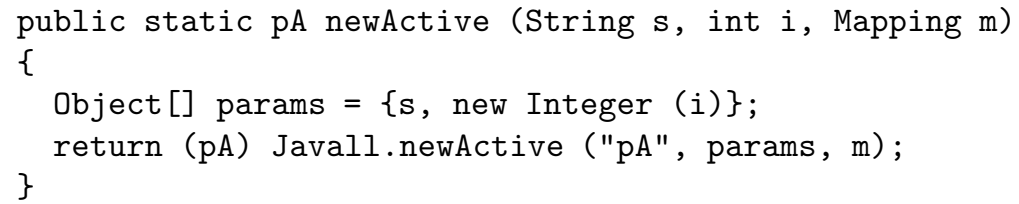

As another important advantage, this technique reduces the amount of code needed to instantiate active objects using Java//:

$\mathrm{a}=\mathrm{pA}$. newActive ("foo", 7);

\subsubsection{Inter-object synchronization}

Asynchronous message-passing would not be of much interest if the user had to explicitly add synchronization to the code that invokes methods on active objects. Fortunately, Java// provides a mechanism of transparent futures.

When a method is invoked on an active object, it immediately returns a future object. This object acts as a placeholder for the result of the not-yet-performed method invocation. As a consequence, the calling thread can go on with executing its code, as long as it does not need to invoke methods on the returned object, in which case the calling thread is automatically blocked if the result of the method invocation is not yet available.

Future objects in Java// are said to be transparent because they do not require any modification of the caller's code. They are automatically created when a method is invoked on an active object: this is the wait-by-necessity principle[10,12]. Transparent future objects are possible because the automatically created future object is actually an instance of a 
subclass of the returned object, which is compliant with all compile- and runtime type checks and does not weaken software quality.

We believe asynchronous calls and future objects can dramatically improve performance of Internet-wide computations. Because huge latency is the plague of today's Internet, wait-by-necessity can help to automatically overlap computations and communications. As a consequence, the Java virtual machine that runs at a node in a computation spends less time in the idle state waiting for some remote computation to complete.

There are a few cases where future objects are not available. Primitive types cannot lead to future objects because they are not standard objects and thus cannot be subclassed. The same limitation applies to final classes, which includes all arrays.

We have also chosen to forbid the use of future objects for methods that throw checked exceptions. If this were allowed, the execution of a method on an active object could throw an exception in the calling thread at a point where the calling thread has exited the try clause. This would result in an exception being thrown in a context where it cannot be caught, thus modifying the semantics of the application and most likely resulting in an application crash.

We have seen that when using wait-by-necessity, a thread is automatically suspended if it needs an object that is not yet available. This is why wait-by-necessity is said to be a data-driven synchronization mechanism, as opposed to a control-driven synchronization. Yet, there are some situations where synchronization is not directly connected to the call of a method on an object. This is why Java// provides the Javall. waitFor (Object obj) static method which performs an explicit wait on object obj, without calling any method on this object. Another static method, Javall. is Awaited (Object obj), returns a Boolean that indicates if object obj is awaited or not. This enables the thread to do something useful instead of being suspended while obj is awaited.

\subsubsection{Mapping active objects to nodes}

Hiding platform-specific details from the programmer is one of the great strengths of Java. Yet, this fundamental design choice sometimes prevents the programmer from fine-tuning parameters like performance or distribution, because it does not allow any deep insight on what the actual platform is. More specifically, it influences the way Java// deals with mapping active objects to actual processing resources (processors).

Java// library provides a unique abstraction for representing processing power resources: the class Mapping. This is the only locator used for specifying where to create active objects. Since the programmer is entirely free to instantiate these Mapping objects wherever he or she wants, having a single locator class does not mean less flexibility. A Mapping object may, for example, represent a multiprocessor machine where a single JVM transparently maps its threads onto the set of available processors. On the other hand, there may be different Mapping objects within one single VM, so that it is possible to test a distributed application on a single workstation, thanks to the seamless transition between multithreaded and distributed applications which Java// provides. When working with a real cluster of workstations, the programmer may instantiate one Mapping object for each workstation. In the case of a metacomputing application, a Mapping object would be instantiated in every Web browser that participates.

As a consequence, the mapping of active objects to actual computing resources through 
the Mapping class enables the distribution of the application over a set of abstract nodes. Switching between different execution environments (such as a single workstation, a multiprocessor machine, a cluster of workstations or a collection of Web browsers) becomes a matter of only a few seconds, as demonstrated by the examples in the next Section. It also allows for easily implementing any load-balancing policy, either generic or applicationspecific.

\subsection{Intra-object synchronization}

Active objects instantiated through the Javall newActive static method are transparently given their own thread that executes invoked methods with a default FIFO order. This thread is started by the object that owns the queue of pending method invocations on an object: the body.

Java// provides a mechanism for specifying synchronization of method invocations on a given active object. The purpose of this mechanism is to enhance the standard thread synchronization mechanism[13] with an explicit method for specifying synchronization. The biggest difference with the standard thread synchronization mechanism is that synchronization is now centralized in one special method of a class, instead of being disseminated in all methods of a class.

The responsibility for specifying the synchronization policy for a class is placed on its live(Body myBody) method. If no live method is provided by the class of the reified object, the body uses its own default live method. For most bodies, the default policy is FIFO.

If the class implements Active, the default mechanism, a thread of control is explicitly available and it is then the responsibility of the live method to explicitly manage the queue of pending requests, if the programmer wishes to override the default FIFO policy. It does so by invoking methods on the Body, such as serveOldest, serveOldest (Method met), serveOldestBut (Method met), waitARequest(). These methods are provided as a service library for managing the queue of pending calls. This service library also includes iterators for safe traversing of the queue of pending requests with access to the effective value of the parameters of the calls.

The FIFO behavior provided by default is simply implemented as follows:

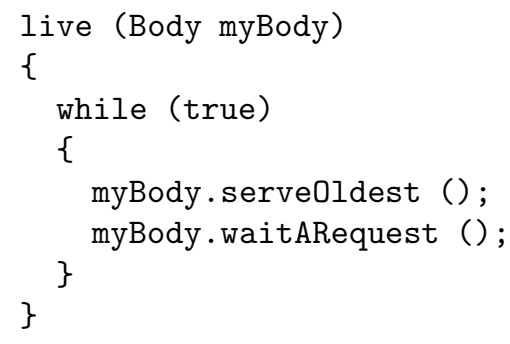

Please note that waitARequest blocks if the state of the queue of pending requests is modified (no active wait).

Now consider the case of the canonical Bounded Buffer example. We assume we have a class FixedBuffer that implements a fixed-length buffer and features methods put and 


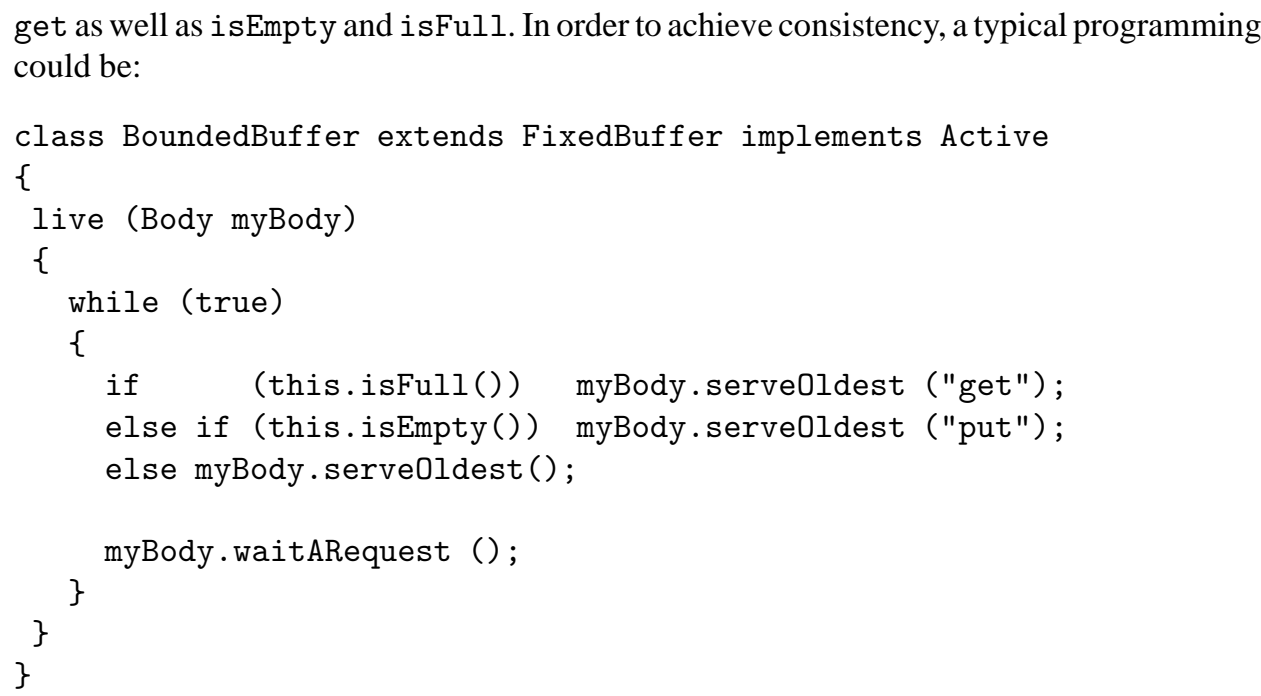

Given these two synchronization constraints, the Body object manages the queue of pending requests properly. As several methods in the same class may have the same name and different parameter types, we provide a convenience mechanism of shortcuts that associates a string to a given method, which results in less code for constraints declaration. If, for example, class A contains two methods foo with different argument types, shortcuts may be created as follows:

java.lang.reflect.Method method1, method2;

// Some code for obtaining Method objects for

// these two 'foo' methods through Reflection API.

$/ /[\ldots]$

Javall.setShortcut ("A", "foo1", method1);

Javall.setShortcut ("A", "foo2", method2);

\subsection{Abstraction for implicit synchronization}

In the case of an implicit synchronization declaration (which is implemented on top of the basic explicit mechanism), the programming of the buffer synchronization policy would be as follows:

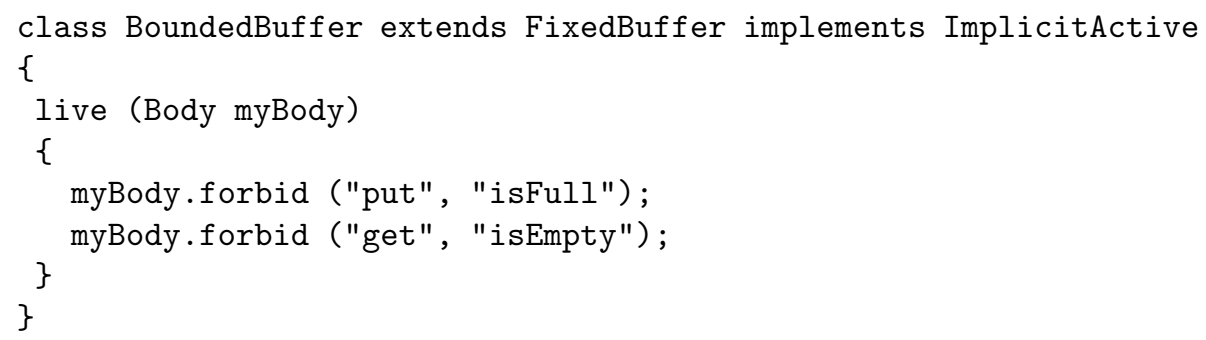




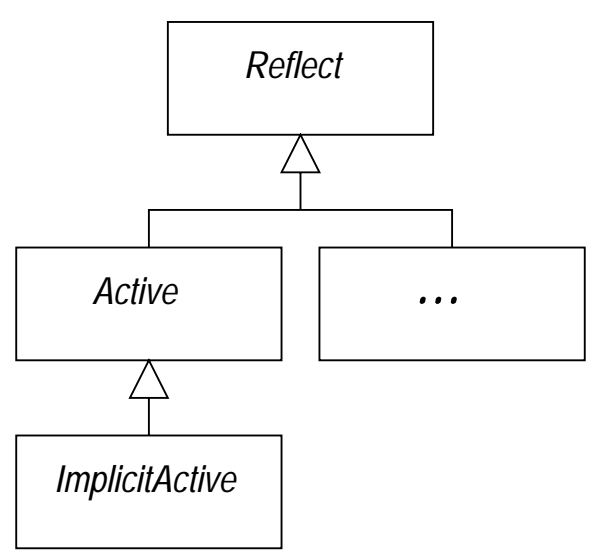

Figure 3. Javal/ interfaces for distribution and synchronization

Implicit synchronization has proven to be better than its explicit counterpart with respect to ease of reuse and better scalability. On the other hand, the overhead needed to decide which call to execute given a set of constraints may not be negligible, and in general the explicit technique has more expressiveness. However, high-performance computing often relies on relatively simple synchronization policies.

This technique is easily extensible and the programmer is free to implement new abstractions for intra-object synchronization[14]. Each of these implementations should result in a body class that implements the synchronization policy and an interface inheriting directly or indirectly from Active, which declares the name of the proxy class (usually the default asynchronous proxy) and the name of the body class (see Figure 3). Such an interface helps organize synchronization abstractions logically and is used by classes such as $\mathrm{pA}$ (see Section 2.2) in order to choose which synchronization technique they would like to use. The interface Reflect does not provide any functionality but acts as a common root interface for all behaviors implemented using the Java// metaobject protocol.

\subsection{A method for reuse}

As Java// is an extension of Eiffel//[12] and C++// [15], it may be the support for a method for reuse first described in[10]. Its main feature is to postpone the identification of active objects in the design of an application. The programmer may then concentrate on application design and not mix it with the division of the application in concurrent activities. The main steps of this method are shown in as follows:

1. sequential design and programming

2. active objects identification

- initial activities

- shared objects

3. active objects programming

- define each active object class 
- define the activity (live)

- use the active objects classes

4. adaptation to constraints

- refine the topology

- define new active objects.

\section{EXAMPLE AND PERFORMANCES}

\subsection{Distributed matrix-vector product}

We have implemented an example proposed by Raje et al. in [16]: a matrix-vector product, the rows of the matrix being split between two machines. The matrix is a square matrix of size 1000 containing float numbers.

We make extensive use of wait-by-necessity in order to automatically overlap local and remote calculations. The time we consider includes sending the vector, performing the calculation and returning the result. It does not include the initial transmission of the remote rows of the matrix. Here is the code for the main method of the sequential version of the program:

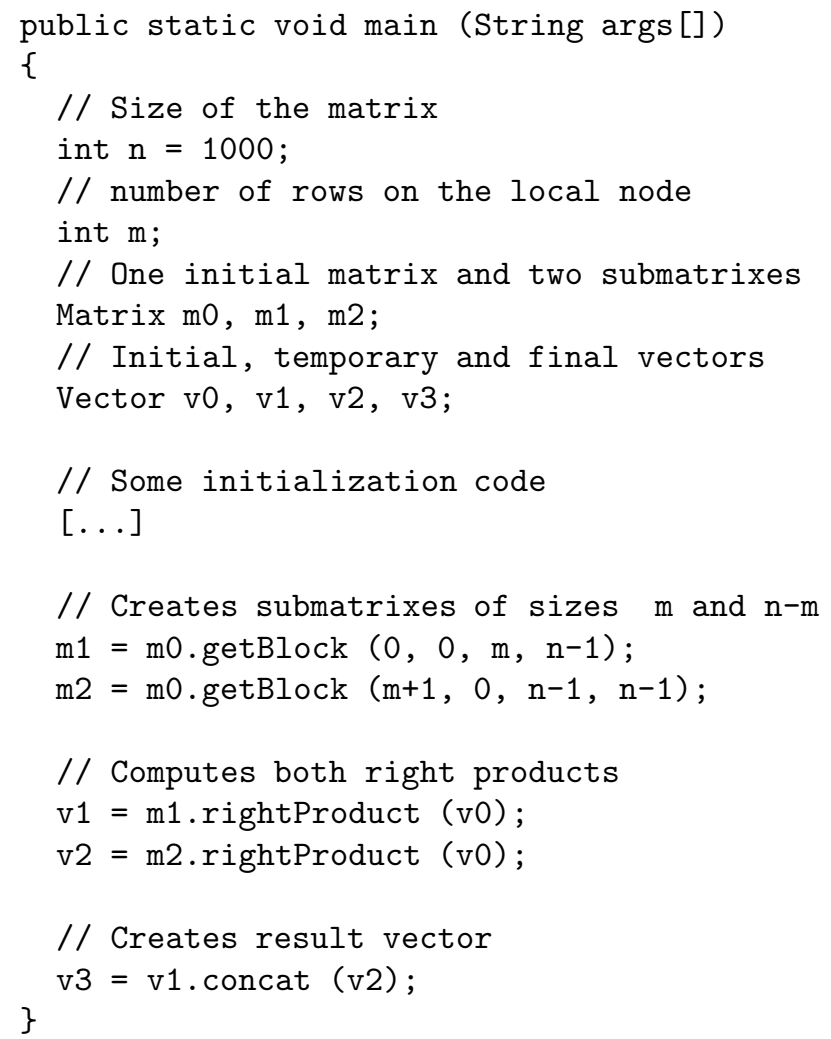


Now assume we want to get a multithreaded and distributed version of this program. The only modifications we need to bring to the source code are located in the portion of code where we create the objects we want to make active.

$\mathrm{m} 1=\mathrm{m} 0 \cdot \operatorname{getBlock}(0,0, \mathrm{~m}, \mathrm{n}-1)$;

$\mathrm{m} 2=\mathrm{m} 0 \cdot \operatorname{getBlock}(\mathrm{m}+1,0, \mathrm{n}-1, \mathrm{n}-1)$;

If we had access to the code of the Matrix class, we would like to modify it in such a way that the implementation of method getBlock in class Matrix now returns an active object instead of a standard one. But this method would have several flaws:

- Every invocation of this method would return an active object, even if we do not want it to.

- Its signature would have to be modified in order to take into account a new argument: the Mapping object specifying where to create the active object.

This is why we provide the Javall.turnActive method in order to attach an active behavior to an active object after its creation. As a consequence, we only need to add these two lines to method main, after the standard $\mathrm{m} 1$ and $\mathrm{m} 2$ :

m1=(Matrix) Javall. turnActive (m1, remoteMapping);

$\mathrm{m} 2=($ Matrix) Javall. turnActive (m2, localMapping);

As a general rule, we do not assume we have access to the code of the linear algebra library. Consequently, using Javall . newActive is not always possible, since submatrices might be instantiated inside the library (actually inside the body of method getBlock) and returned as a result of this method invocation.

Parallelism is achieved here because, as both $\mathrm{m} 1$ and $\mathrm{m} 2$ are active objects, both calls to rightProduct are asynchronous and return future objects for representing the not-yetavailable result vectors (namely v1 and v2). As a consequence, the thread that executes main launches these two products and is then blocked on the call to concat because $v 1$ is not available at the moment. Both products are executed in parallel on two different nodes of the computation (the local node localMapping and a remote one designated as remoteMapping.

Let us now assume we want to run the same program on an SMP machine with a JVM using native threads. The only modification needed would be to change remoteMapping to the current node (localMapping here), through the javall-mapping file.

\subsection{Performances}

Figure 4 shows the time needed to compute the product with respect to the number of rows on the remote machine. Both the local and the remote machine were UltraSparcs. As in [16], the minimum is reached for 400 remote rows and 600 local ones. This is not surprising at all since both Java// and ARMI are implemented on top of RMI.

Our implementation of Java// is based on Java RMI. Our experience with RMI lead us to the following conclusion: RMI shows catastrophic performance when it comes to exchanging large-size messages, such as a whole matrix or a very large graph of objects. RMI itself is not directly responsible for this, but the default serialization mechanism is. As a general rule, it is hard to achieve speedup on a network of workstations when the communication/computation ratio is too high. 


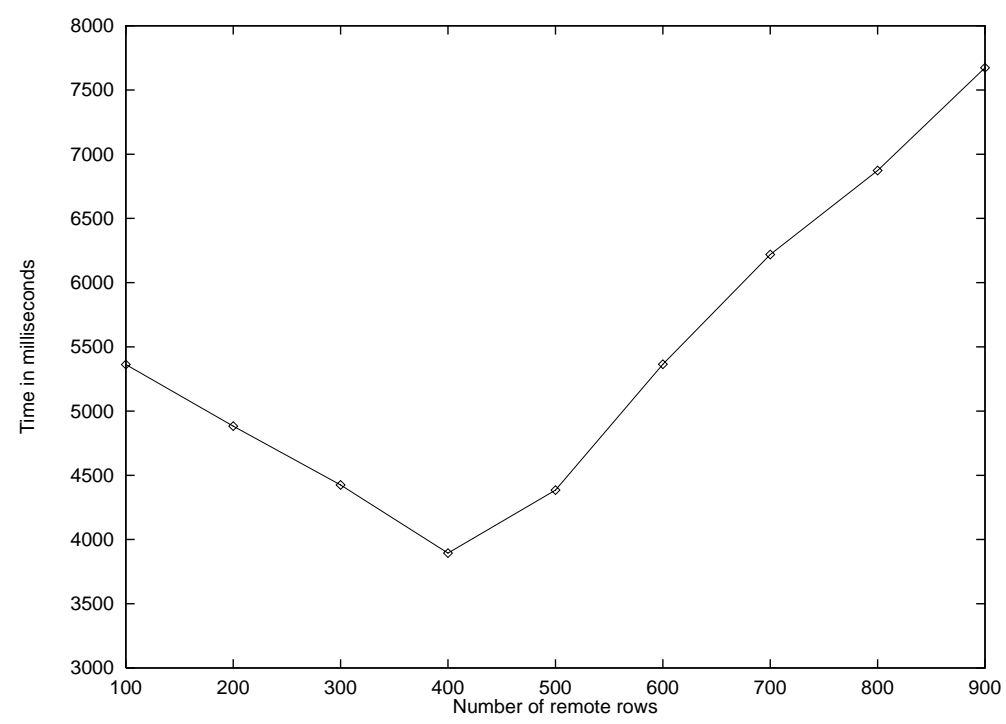

Figure 4. Javall performances for matrix-vector computation

\subsection{A parallel and distributed collaborative application}

\subsubsection{Introduction}

In order to demonstrate the power of Java// regarding synchronization, we have implemented a collaborative application. It showcases three of the major benefits of Java//: ease of distributed programming, speedup through parallel calculation, and ease of synchronization. Several users can collaboratively view and manipulate a 3D scene, which involves election and synchronization issues. The image for the scene is calculated by a dynamic set of rendering engines using a raytracing algorithm, everything being controlled by a central dispatcher (see Figure 5).

Users can rotate the scene and send messages to other users. They are informed by the dispatcher on events of public interest (i.e. user set changes, elections, rendering).

\subsubsection{Design}

An instance of class Dispatcher (see Figure 6) acts as the central management module. It uses Java// to dynamically distribute the calculation on remote hosts, to centralize all synchronizations and to provide collaborative services to the users. Furthermore, it serves as a static entry point for the users (each running an instance of UserFrame), it manages the set of users and holds all the cross-references.

The first user to register at the Dispatcher instance determines the scene to be rendered. The rendering task is split up by the Dispatcher in a stack of image Interval instances. Those are assigned to the set of RenderingEngine instances with a first-returned, nextassigned load-balancing. Each RenderingEngine receives one initial Interval first, the remainder of the Interval stack is given to the engines when they return the results. The Dispatcher collects the image data and forwards it to the user's UserFrame instance. 


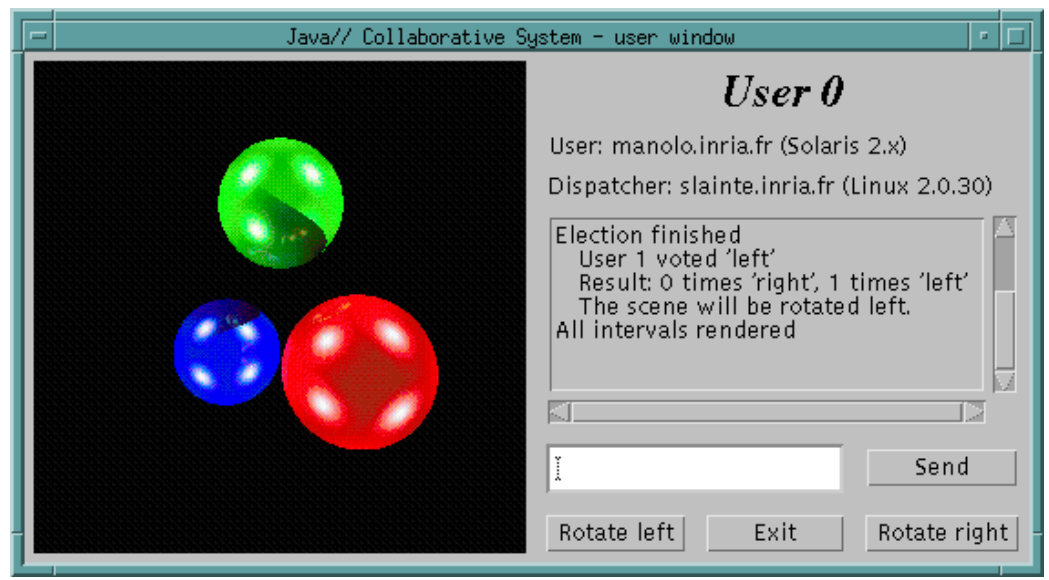

Figure 5. Screen shot of a user window

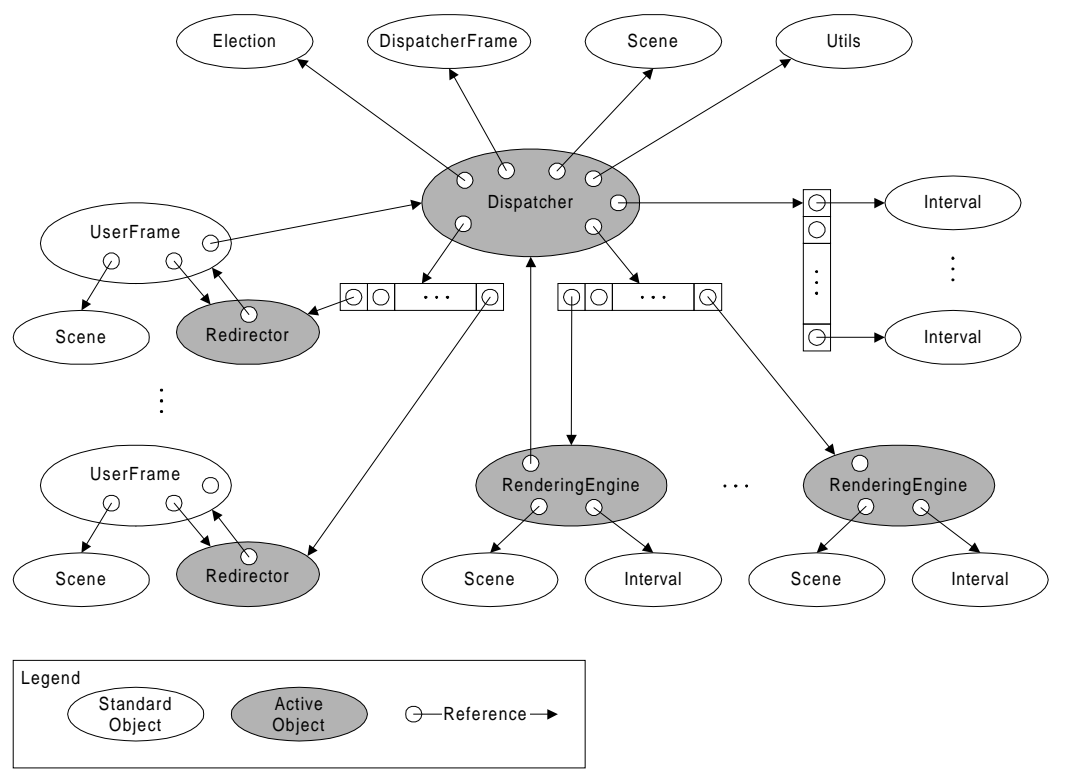

Figure 6. Runtime instance graph

Thanks to Java//, all method calls between the application modules are asynchronous, which provides efficient communication-calculation overlapping. Neither the users nor the Dispatcher is waiting for the calculation results; they are asynchronously called back instead.

UserFrame and DispatcherFrame inherit from the AWT Frame, which is a heavyweight class with a lot of public methods. To keep the stub small and to minimize overhead, we choose to activate Redirector and Dispatcher instead. 
Note that each active object can be selectively and dynamically mapped into a Java virtual machine (JVM), which can be either local or remote. This gives a wide range of distribution options, from having all active objects in the same local JVM, to having each active object distributed in its own remote JVM. The standard objects always reside in the JVM of the active object they are controlled by.

\subsubsection{Synchronization}

All the Dispatcher synchronizations are centralized in the live method. It uses Java// service methods (see Section 2.3) to manipulate the queue of pending method calls.

An instance of Election is created when two or more users are registered at the dispatcher and one user clicks to rotate in any direction. This request is converted into the first vote. The new Election instance starts its own standard Java thread, notifies all users of the election, and runs until either all users have voted or a $5 \mathrm{~s}$ time-out is reached. It then counts the votes, picks the decision (either unanimity or majority mode) and starts the rotation. While the election is running, rotate requests are taken as votes, and other requests are ignored. While the image is rendered, rotate requests are refused, and other requests are served.

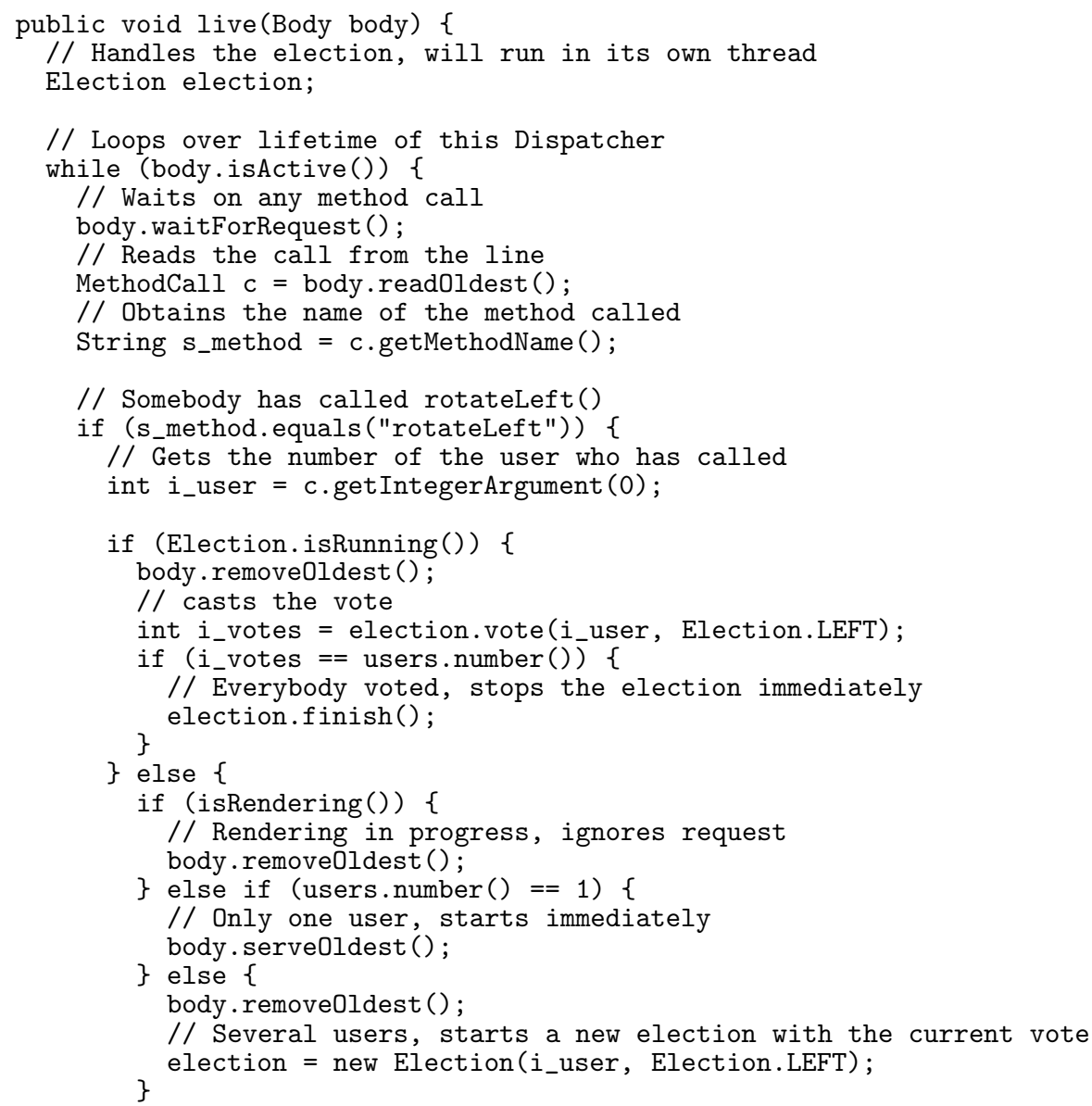




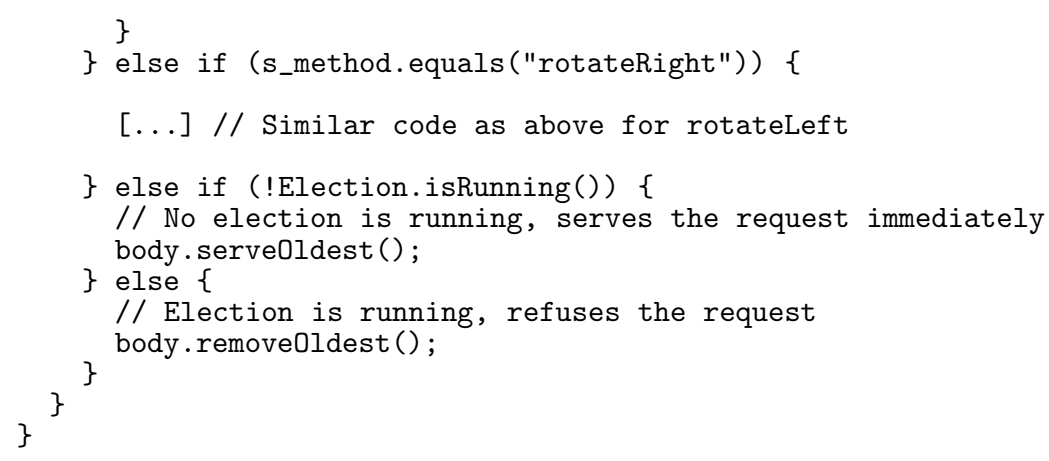

\section{IMPLEMENTATION: A META-OBJECT PROTOCOL}

Java// is built on top of a metaobject protocol (MOP)[17] that permits reification of method invocation and constructor call. As this MOP is not limited to the implementation of our transparent remote objects library, it also provides an open framework for implementing powerful libraries for the Java language.

As for any other element of Java//, this MOP is entirely written in Java and does not require any modification or extension to the Java Virtual Machine, as opposed to other metaobject protocols for Java[18]. It makes extensive use of the Java Reflection API, thus requiring JDK 1.1 or higher. JDK 1.2 is required in order to suppress default Java language access control checks when executing reified non-public method or constructor calls.

If the programmer wants to implement a new metabehavior using our metaobject protocol, he or she has to write both a concrete (as opposed to abstract) class and an interface. The concrete class provides an implementation for the metabehavior he or she wants to achieve while the interface contains its declarative part. The concrete class implements interface Proxy and provides an implementation for the given behavior through the method reify:

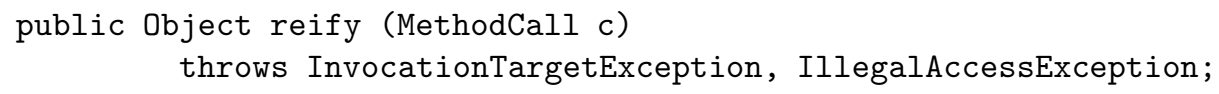

This method takes a reified call as a parameter and returns the value returned by the execution of this reified call. Automatic wrapping and unwrapping of primitive types is provided. If the execution of the call completes abruptly by throwing an exception, it is propagated to the calling method, just as if the call had not been reified.

The interface that holds the declarative part of the metabehavior has to be a subinterface of Reflect (the root interface for all metabehaviors implemented using Java//). The purpose of this interface is to declare the name of the proxy class that implements the given behavior. Then, any instance of a class implementing this interface will be automatically created with a proxy that implements this behavior, provided that this instance is not created using the standard new keyword but through a special static method: MOP.newInstance. This is the only required modification to the application code. Another static method, MOP .newWrapper, adds a proxy to an already-existing object; the turnActive function of Java//, for example, is implemented through this feature.

Here is the implementation of a very simple yet useful metabehavior: for each reified call, the name of the invoked method is printed out on the standard output stream and 
the call is then executed. This may be a starting point for building debugging or profiling environments:

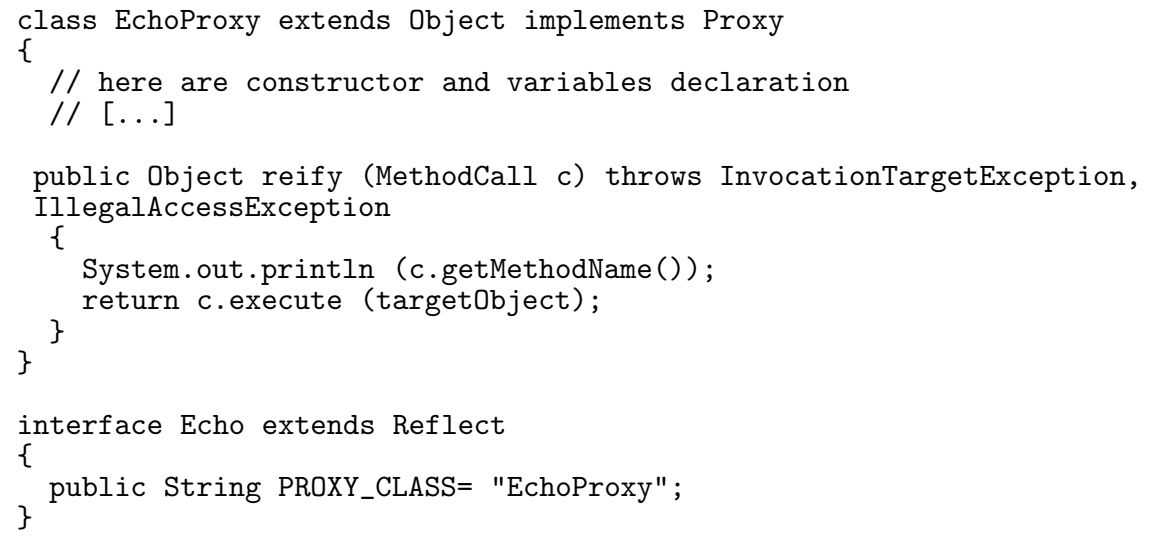

Instantiating an object of any class with this metabehavior can be done in three different ways: instantiation-based, class-based or object-based. Let us say we want to instantiate a Vector object with an Echo behavior:

- Standard Java code would be:

Vector $\mathrm{v}=$ new Vector (3);

- Java// code, with instantiation-based declaration of the metabehavior:

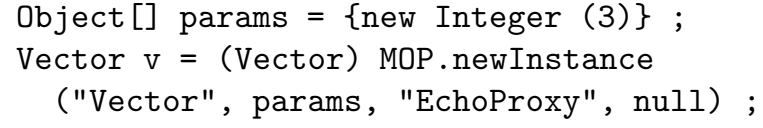

The last parameter is because we do not have any additional parameter to pass to the proxy.

- with class-based declaration:

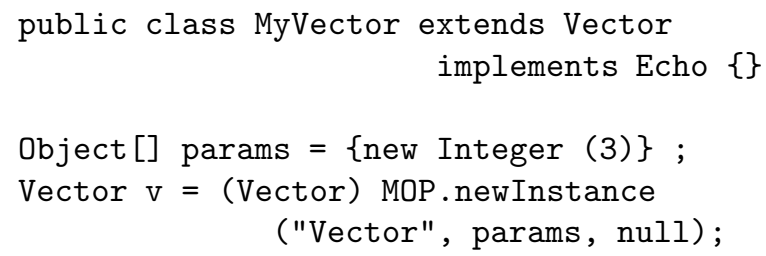

- with object-based declaration:

Vector $\mathrm{v}=$ new Vector (3); $\mathrm{v}=($ Vector) MOP.newWrapper ("EchoProxy", v);

which is the only way to give a metabehavior to an object that is created in a place where we cannot edit source code. A typical example could be an object returned by a method that is part of an API distributed as a JAR file, without source code. Please note that, when using newWrapper, the invocation of the constructor of the class Vector is not reified. 


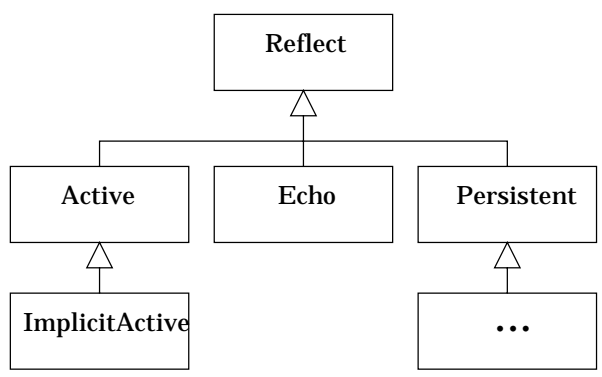

Figure 7. Javal/ interfaces

All the interfaces used for declaring metabehaviors inherit directly or indirectly from Reflect. This leads to a hierarchy of metabehaviors such as shown in Figure 7. Note that ImplicitActive inherits from Active to highlight the fact that implicit synchronization somewhere always relies on some hidden explicit mechanism. Interfaces inheriting from Reflect can thus be logically grouped and assembled using multiple inheritance in order to build new metabehaviors out of existing ones.

Due to its commitment to be a $100 \%$ Java library, the MOP has a few limitations:

- Calls sent to instances of $f$ inal classes (which includes all arrays) cannot be reified.

- Primitive types cannot be reified because they are not instances of a standard class.

- Final classes (which includes all arrays) cannot be reified because they cannot be subclassed.

\section{CONCLUSION AND FUTURE WORK}

We have designed and implemented Java//, a 100\% Java library aimed at providing a framework for the seamless development of sequential, multithreaded and distributed applications. It features transparent active and remote objects as well as asynchronous calls, transparent future objects and wait-by-necessity. The most important feature of Java// is that it provides very smooth transition between sequential, multithreaded and distributed programming.

Java// is implemented without any modification of the Java Virtual Machine or any element of the standard Java environment. It is only made of $100 \%$ Java classes and heavily relies on Java Reflection API and Java RMI.

We are currently working on a new implementation of Java// which will take advantage of new JDK 1.2 features (suppression of language access control checks, Reflection and RMI enhancements, weak references, ...) as well as take into account deprecated parts of the thread API.

We are also working on an implementation of the Salishan problems[19] as a test bed.

$\mathrm{Java} / /$ is available for downloading along with source code, documentation and examples at http://www.inria.fr/sloop/javall.

\section{REFERENCES}

1. C. Catlett and L. Smarr, 'Metacomputing', Commun. ACM, 35, 144-152 (1992). 
2. I. Foster and C. Kesselman, 'Globus: A metacomputing infrastructure toolkit', Int. J. Supercomput. Appl. High Perform. Comput., 11(2), 115-128 (1997).

3. Geoffrey C.Fox and Wojtek Furmanski, 'Java for parallel computing and as a general language for scientific and engineering simulation and modelling', 1996.

4. Ken Arnold and James Gosling, The Java Programming Language, The Java Series, AddisonWesley, Reading, MA, USA, May 1996.

5. James Gosling and H. McGilton, The Java Language Environment, Sun Microsystems Computer Company, May 1995.

6. C. A. R Hoare, 'Monitors: An operating system structuring concept', Commun. ACM, 10, 549-557 (1974).

7. Jean-Pierre Briot and Akinori Yonezawa, 'Inheritance and synchronization in concurrent OOP', in European Conference on Object-Oriented Programming (ECOOP'87), Springer-Verlag, LNCS 276, 1987, pp. 32-40.

8. W. M. Yu and A. L. Cox, 'Java/DSM: a platform for heterogeneous computing', in ACM 1997 Workshop on Java for Science and Engineering Computation, June 1997.

9. Michael Philippsen and Matthias Zenger, 'Javaparty - transparent remote objects in Java', in ACM 1997 Workshop on Java for Science and Engineering Computation, June 1997.

10. Denis Caromel, 'Toward a method of object-oriented concurrent programming', Commun. ACM, 36(9), 90-102 (1993).

11. Erich Gamma, Richard Helm, Ralph Johnson and John Vlissides, Design Patterns - Elements of Reusable Object-Oriented Software, Professional Computing Series, AW, 1995.

12. Denis Caromel, 'Service, asynchrony, and wait-by-necessity', J. Object-Orientated Program., 12-22 (1989).

13. Doug Lea, Concurrent Programming in Java: Design Principles and Patterns, Addison-Wesley Java series. Addison-Wesley, Reading, MA, USA, November 1996.

14. Denis Caromel, 'Programming abstractions for concurrent programming', in Technology of Object-Oriented Languages and Systems, PACIFIC (TOOLS PACIFIC '90), November 1990.

15. Denis Caromel, Fabrice Belloncle and Yves Roudier, The $C++/ /$ System, MIT Press, 1996.

16. Rajeev R. Raje, Joseph I. William and Michael Boyles, 'An asynchronous remote method invocation (armi) mechanism for Java', in ACM 1997 Workshop on Java for Science and Engineering Computation, June 1997.

17. Gregor Kiczales, Jim des Rivières and Daniel G. Bobrow, The Art of the Metaobject Protocol. MIT Press, 1991.

18. Juergen Kleinoeder and Michael Golm, 'Metajava: An efficient run-time meta architecture for java', Tech. Report TR-I4-96-03, Univ. of Erlangen-Nuernberg, IMMD IV, 1996 (English).

19. John T. Feo, 'A comparative study of parallel programming languages: the Salishan problems', in Special Topics in Supercomputing, Vol. 6, North-Holland Publishing Co., Amsterdam, The Netherlands, 1992. 